\title{
A SPATIAL DATABASE MODEL FOR MOBILITY MANAGEMENT
}

\author{
A. $\operatorname{Ajmar}^{1}$, E. $\operatorname{Arco}^{2}$, P. Boccardo ${ }^{2 *}$ \\ ${ }^{1}$ ITHACA, Via Pier Carlo Boggio 6, 10138 Torino, Italy - andrea.ajmar@ithaca.polito.it \\ ${ }^{2}$ Politecnico di Torino - DIST, Torino, Italy - (emere.arco, piero.boccardo)@ polito.it
}

KEY WORDS: Road Network, Mobility Management, Standards, Spatial Data Model, Traffic Measures

\begin{abstract}
:
In urban and metropolitan context, Traffic Operations Centres (TOCs) use technologies as Geographic Information Systems (GIS) and Intelligent Transport Systems (ITS) to tackling urban mobility issue. Usually in TOCs, various isolated systems are maintained in parallel (stored in different databases), and data comes from different sources: a challenge in transport management is to transfer disparate data into a unified data management system that preserves access to legacy data, allowing multi-thematic analysis. This need of integration between systems is important for a wise policy decision.

This study aims to design a comprehensive and general spatial data model that could allow the integration and visualization of traffic components and measures. The activity is focused on the case study of 5T Agency in Turin, a TOC that manages traffic regulation, public transit fleets and information to users, in the metropolitan area of Turin and Piedmont Region.

The idea is not to replace the existing implemented and efficient system, but to built-up on these systems a GIS that overpass the different software and DBMS platforms and that can demonstrate how a spatial and horizontal vision in tackling urban mobility issues may be useful for policy and strategies decisions. The modelling activity take reference from a review of transport standards and results in database general schema, which can be reused by other TOCs in their activities, helping the integration and coordination between different TOCs. The final output of the research is an ArcGIS geodatabase, which enable the customised representation of private traffic elements and measures.
\end{abstract}

\section{INTRODUCTION}

\subsection{General context}

Mobility management in metropolitan areas mainly concerns the application of strategies and policies to reduce travel demand or to redistribute demand in space and time. This activity has a large impact on the socio-economic growth and in general in the quality of life of citizens. In particular, high quality mobility stimulates territorial cohesion, trades and job creation, increasing the general economy, while low quality mobility generates high levels of congestion, air pollution and noise, increases the risk of accidents and may generates social exclusion.

Between strategies and actions to improve mobility efficiency, a key role is played by Intelligent Transport Systems (ITS), a set of technologies devoted to automatically collect, process and spread traffic trends, providing informative services to users as to Traffic Operations Centres (TOCs) in near real-time. On the other side, GIS provides the framework for integrating road networks and traffic information (Thompson et al., 2019).

European and National policies today stress the role of ITS and GIS in mobility management. In the European context, the need of a core network of strategic European infrastructures emerges, to be achieved through the deployment of large-scale intelligent and interoperable technologies, in order to optimise the capacity and the use of infrastructures (European Commission, 2011). On the other side, the Italian Minister for Infrastructures and Transport, has promoted the Smart Road Initiative (Ministero delle Infrastrutture e dei Trasporti, 2018), which aims to create a technological environment for the interoperability and communication between infrastructures and vehicles, enabling a new mobility user experience and mobility services. In particular, in a positional paper ((Ministero delle Infrastrutture e dei Trasporti, 2018) linked to the initiative, various implementations aspects are described, focusing also on the predisposition of a reference road network (certified by experts), with three level of detail, over which reference static and dynamic characteristics, as data and information about traffic (raw and forecasted).

Both documents underline the need of transport data integration both in terms of information about physical infrastructures and information on the status of the infrastructure (level of service, traffic flow, timetables...), in particular between administrative levels.

The creation of an integrated and efficient mobility system represents indeed the solution for organizing and monitoring transport across different modes, in order to increase the sustainable mobility through the creation of new opportunities for collective mobility, enhancing TOCs supervision capacity and management efficiency, and users' accessibility to mobility.

\subsection{Structure of the study}

This study starts from the case study of 5T Agency in Turin, a TOC that manages traffic regulation, public transit fleets and information to users, in the metropolitan area of Turin and Piedmont Region.

In particular, the agency has set up during years a wide system of Intelligent Transport Systems (ITS) technologies that acquires continuously measures and traffic information, which are used to deploy information services to citizens and public administrations. However, the spatial nature of these data is not fully considered in the daily operational activity, with the result of difficulties in information integration. Indeed, the agency lacks a complete GIS solution that includes all the management information in an organized spatial and "horizontal" vision.

The main research question concerns the integration of different kind of data in a unique GIS spatial data model. Spatial data interoperability is critical and particularly challenging in the transport field because geographic data definition in legacy database can vary widely: different data format and standards, 
data inconsistencies, different spatial and temporal granularities, different methods and enforcing rules that relates measures, events with physical infrastructures.

A review of existing standards in transportation field, focused on the spatial data aspects, allowed to build a common reference over which building a new proposal. Standards are analysed and compared, looking at their main purpose, at their topological and geometrical aspects, at the completeness of features definition, and at the strategies adopted for temporal management.

The first output is a logical level spatial data schema which can enable the fulfilment of multiple tasks over a single network reference data. The solution is applied to the real context of 5T Torino agency, where the physical model is implemented through an ArcGIS geodatabase, tailored on the specific agency needs.

The logical schema is also proposed as possible solution to set up an homogeneous road network in the context of the URBAN-GEO BIG DATA (URBAN GEOmatics for Bulk Information Generation, Data Assessment and Technology Awareness), a Project of National Interest funded by the Italian Ministry of Education that aims at contributing to the exploitation of heterogeneous geodata sources such as VGI, geo-crowdsourcing, earth observation, etc. for a better understanding of urban dynamics.

\section{MODELLING ACTIVITY}

\subsection{Road network abstraction}

The modelling activity concern the definition of a network model, which are the most prominent models used in transport field, thanks to their simplicity, elegant form and functionality, which allow to represent the flows of passengers, cars, freight moving through the network's lanes (Thill, 2000). A network is a type of mathematical digraph that captures relationships between objects using connectivity. The connectivity may or may not be based on spatial proximity and is usually guaranteed by the application of topology rules. The network model contains logical information such as connectivity relationships among nodes and links, directions (or navigability) of links, and costs (or impedances) of nodes and links. This information is critical in routing applications and spatial analysis, as they reflect the real-world characteristics of transport networks such as one-way streets.

However, as streets in reality are areas, there are multiple ways to model a street. A common abstraction uses logical centreline or physical centreline. Logical centreline represents the approximate midpoint of the street across its width, in order to show the general location and shape of the facility at a relatively small scale. Physical centreline usually represents the approximate midpoint of carriageway across its width and are most used at bigger scale. Similarly, intersections also can have different levels of abstraction and their representation is dependent from centreline abstraction used.

Choosing an appropriate level of abstraction that cope with the application purpose and manageable complexity is never a trivial task. The simplest approach to making a transport dataset is to create independent segments representing linear portions of the total system (Butler, 2008; Rodrigue, 2017). A typical way to do this is to segment longer facilities at intersections. Each segment gets the same set of attributes so that values extending across a road are duplicated for all segments to which the value applies. Some database designers may argue that this simple design is inefficient, but it is indeed the most used in transport databases (Butler, 2008).

The described approach allows to model the static information of a road network; however, transport data comprises also a series of dynamic data: mean flows and speeds of vehicles traversing segments, accidents and roads works which can limit the navigability of an edge, between many others. Between approaches for modelling dynamic transport data, the use of linear referencing systems is one of the most used. A linear referencing system (LRS) can be defined as "a system of determining the position of an entity relative to other entities to some external frame of reference" (Goodwin et al., 1998). A linear referencing method (LRM) is the set of technical processes used to determine, specify, and recover a location within an LRS. It includes rules for managing the measurement process, as how to define the origin, the unit of measure, the precision and scale for measured distances (Figure 1).

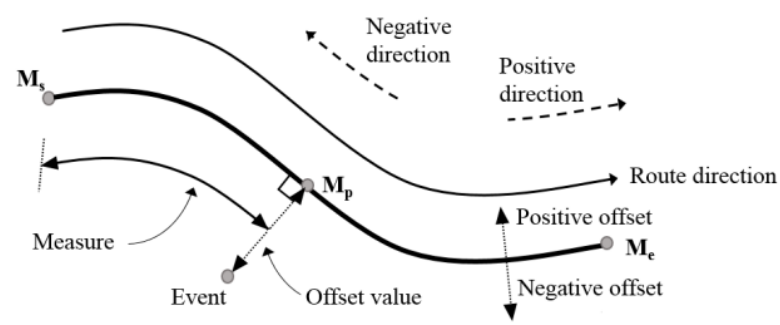

Figure 1. Linear Referencing and Dynamic segmentation.

Dynamic segmentation is the software-based process of transforming linearly referenced data stored in a table into a virtual (on-the-fly) feature that can be displayed on a map (Cadkin, 2002 a, b).

The use of LRM within a defined LRS, in combination with dynamic segmentation allows to significantly reduce the amount of disk space needed to store data, which is particularly critical for this type of information which can have high rates of updates. On the other side, as different LRS exists, interoperability between systems is usually problematic (Butler, 2008, Goodwin et al., 1998).

\subsection{Requisites of the database}

Starting from 5T Agency needs, a series of requirements for the spatial database have been defined.

In particular, the agency is responsible of many tasks comprising info mobility services (both for public and private transport) as well as traffic management policies (re-routing, traffic light management and priorities). This framework has led to a complex situation on the data management side, were multiple databases, specialised in different tasks, cannot be spatially integrated without a huge effort.

Within this complex framework, the first requisite was to build a spatial database able to integrate different components devoted to different task.

At the static information level (road network, public transport stops and routes, ancillary traffic objects as cameras, traffic lights, gates, informative panels...) the goal was to allow the visualisation of the positions over a common network reference. Dynamic information related to static elements is also considered: using the common reference road network set up, the database structure had to enable the visualisation of different traffic measures (flows, speeds, travel times...), referred to single point of measures as well to stretch of roads. From a temporal point view, the database must allow to 
visualise single instants as well time range and time series. These measures will be then used within network analysis algorithm in order to produce accessibility analysis, services areas, routing solution using real data coming from sensors as impedances for the model.

\subsection{Overview of existing transport standards}

Standardization is a process that aims to help in technological development and to share good ideas and solutions, through documenting practical information, best practices and agreed solutions on global problem (ISO, 2017). Thanks to standardization initiatives promoted from 1995 in the contest of spatial data handling, significant technological development has made. In the context of this research, standards represent the main source of reference, documenting the state of art of the spatial data modelling activity.

As a first step, relevant standards in the field of GIS and transport are deepened and compared. Between existing standards, the ones considered in this research are presented in Table 1, subdivided between their main purposes.

\begin{tabular}{|c|c|c|c|}
\hline & $\begin{array}{l}\text { Road Network } \\
\text { Representation }\end{array}$ & $\begin{array}{c}\text { Public Transport } \\
\text { Network } \\
\text { Representation }\end{array}$ & $\begin{array}{l}\text { Dynamic data } \\
\text { Representation }\end{array}$ \\
\hline $\begin{array}{l}\text { INSPIRE Road } \\
\text { Network Model }\end{array}$ & $\mathrm{X}$ & & \\
\hline $\begin{array}{l}\text { O\&M INSPIRE } \\
\text { implementation }\end{array}$ & & & $\mathrm{X}$ \\
\hline $\begin{array}{r}\text { FGDC - } \\
\text { Transportation } \\
\text { Data model }\end{array}$ & $\mathrm{X}$ & $\mathrm{X}$ & $\mathrm{X}$ \\
\hline CityGML & X & & \\
\hline TRANSMODEL & & $\mathrm{X}$ & $\mathrm{X}$ \\
\hline GTFS & & $\mathrm{X}$ & $\mathrm{X}$ \\
\hline TMC & $\mathrm{X}$ & & \\
\hline DATEX II & & & $\mathrm{X}$ \\
\hline S.I.MO.NE & & & $\mathrm{X}$ \\
\hline
\end{tabular}

Table 1. Overview of standards considered for the research, categorised by their main purpose.

Data models have been compared from the point of view of the segmentation model (the choice of how to represents roads and their connection, and the related physical structures), deepening geometrical and topological aspects, from the semantic point of view (the meaning of the different terms used to describe physical object), from the point of view of temporal aspects, in order to take into account ways to represents dynamic aspects as measures and events.

Looking at the aspects of road spatial visualisation and thematic representation, the INSPIRE Road Network Model (INSPIRE, 2013), the FGDC Model (Federal Geographic Data Committee, 2008) and the CityGML are the most complete: their definition allows a detailed geometrical representation and the richness of attributes enables a variety of thematic representations (road hierarchy, speed limits, lanes, ...). On the other hand, Traffic Message Channel (TMC) standards is defined only at logical level (points and relation between points), without a precise geometrical representation of the roads and with a good support for road naming only (Arco et al., 2017). TRANSMODEL (TRANSMODEL, 2015) and GTFS, also allow a precise geometrical representation of the public transport routes (even if in GTFS the geometrical definition of a linear element is optional).
The connectivity rules, which enable routing over the network, are well defined in the INSPIRE and FGDC model, where a robust definition of the relationships between points, lines and groups of elements exists. In particular, a strength of FGDC model is the solid integration between public transport network and the infrastructure over which they insist, defined by specific topological relationships. The CityGML has a definition for areal routing but is poorly supported by real cases. In addition, the support for LRM is present in the FGDC model, where events are well defined and integrated in the schema. LRM support is less developed for the INSPIRE model, where indeed there is a lack in events definition. LRM are obviously well supported by info-mobility standards: both DATEX and S.I.MO.NE (Arneodo et al., 2009) models comprise references for TMC linear referencing, as well OpenLR system and WGS 84 coordinates.

\begin{tabular}{|l|l|l|}
\hline GTFS & TRANSMODEL & FGDC \\
\hline Agency & Operator/Autority & TransitStop [Owner] \\
\hline Stop & $\begin{array}{l}\text { Scheduled Stop Point, } \\
\text { stop place, tariff zone }\end{array}$ & $\begin{array}{l}\text { TransitStop, } \\
\text { TransferCluster and } \\
\text { Facility }\end{array}$ \\
\hline Route & Line & TransitRoute \\
\hline Trip & Vehicle journey & $\begin{array}{l}\text { Trip (temporal aspect), } \\
\text { Pattern (spatial aspect) }\end{array}$ \\
\hline Stop Times & $\begin{array}{l}\text { Passing times, stop point } \\
\text { in journey pattern, } \\
\text { distance in route link }\end{array}$ & Time Point \\
\hline Calendar & $\begin{array}{l}\text { Day type, period and day } \\
\text { of week }\end{array}$ & - \\
\hline Calendar dates & Operating day & - \\
\hline Fares attributes & Fare element price & Fare, FareType code list \\
\hline Fare rules & $\begin{array}{l}\text { Fare element, distance } \\
\text { matrix }\end{array}$ & $\begin{array}{l}\text { FarePolicyType code } \\
\text { list, FareType code list }\end{array}$ \\
\hline Shape & Route, LinkProjection & TransitPath \\
\hline Frequency & (frequency) & - \\
\hline Transfer & $\begin{array}{l}\text { Connection (link), service } \\
\text { journey interchange, } \\
\text { service journey pattern } \\
\text { interchange, default } \\
\text { interchange }\end{array}$ & ConnectionSeg \\
\hline
\end{tabular}

Table 2: GTFS tables and equivalent classes of Transmodel and FGDC Transit Framework. Readapted from Knowles and Miller, 2008.

From the semantic point of view, data dictionaries have been deepened: the existence of code lists and related definitions has been considered as a requisite. FDGC, TRANSMODEL and DATEX standards have accurate code lists: in particular the TRANSMODEL is data dictionary is really rich and comprises lots of specific cases, and DATEX comprises a rich set of traffic events types and categories, as well as measurements sites definitions. The main problem observed during the semantic comparison of terms is the high variability in terms definition. In public transport models this is evident, where the vernacular use of terms as route, journey, trip or stop can cover a wide range of overlapping concepts, leading to confusion when information systems and data needs to be compared. TRANSMODEL seems the most accurate with a technical term definition and it has been used as reference to compare the use of different terms in other standards models (see Table 2).

Temporal aspects are poorly defined in INSPIRE, FGDC and CityGML: FGDC comprises a temporal attribute to define the last update of an event, INSPIRE instead comprises the definition of a versioning systems, and CityGML demand the management to specific custom extensions to be built. These components are not enough for a complete management of 
timing component. The Observations and Measurements model, implemented within INSPIRE, may cover the lack of a system for temporal management: it allows to link data with temporal attributes as instants, ranges and time series, which allow a complete definition for temporal management. The weakness of the model is mainly due to the lack of practical implementation for traffic management. Temporal aspects are indeed deepened in the TRANSMODEL as well in info-mobility standards. These models usually relate spatial elements to a temporal definition (a point object to instants and linear objects to ranges), highlighting the strong integration between spatial and temporal data.

\subsection{The conceptual framework}

First activity to develop a conceptual data model regards the clear and unambiguous definition of general elements and relationships that compose the model.

An overview of the conceptual data model is given in Figure 2.

The conceptual model considers several aspects: a basic geometry definition and rules for topology definition from the segmentation point of view, and a basic semantic level, which comprises also ancillary traffic elements, usually not considered in other standards. In addition, it tries to integrate the general road network with elements of the public transport network, and, through the event modelling, temporal measures related to private traffic as public transport monitoring.

The model is based on four abstract classes (red in Figure 2), which can be used as template for more specific ones.

A LINK is a linear and oriented object, which describe the connection between two points. A LINK can be view both as a spatial object with its own geometry, representing a physical element as a carriageway, and as a conceptual object like public transport path or a linear traffic event, with or without a geometry associated.

A POINT is an object used both for the spatial description of the network, and for the description of elements located the network. Therefore, it can be used to represent physical infrastructure objects like intersections, ancillary traffic elements as traffic lights, or more abstract concepts as point traffic events.

A TRANSPORT PROPERTY is used to define a set of characteristics that describe a transport object. These characteristics can change over time; however, the update time is usually low. TRANSPORT PROPERTY is directly related to LINK and POINT classes to which they belong.

An EVENT represents a data directly referred to a LINK or a POINT object. In this class, objects change rapidly over time, differently from TRANSPORT PROPERTY class. It is used mainly to represent raw and estimated traffic measures and traffic events.

From these general elements, aggregations (as sequences and sets, in blue in Figure 2) and specializations (from a functional point of view, in yellow in Figure 3) associations defines more specifics objects, and between them specific relationships are defined.

Point abstract class is specialised in NetworkNode, a point spatial object used for describing connectivity between links, and in PointOnLink, used for the representation of network ancillary elements as traffic lights, traffic monitoring devices and traffic information devices, or other objects that can be located along a link as access points or public transport stops. The association between the NetworkLink and PointOnLink classes exploits the concept of linear referencing: a NetworkLink object can pass through zero, one or multiple PointOnLink object, and reciprocally and a PointOnLink object must be located on a NetworkLink object, at a certain distance from its start point. It has to be noted that this latter relationship is a composite aggregation: if a NetworkLink object is deleted, PointOnLink objects located along it are also deleted.

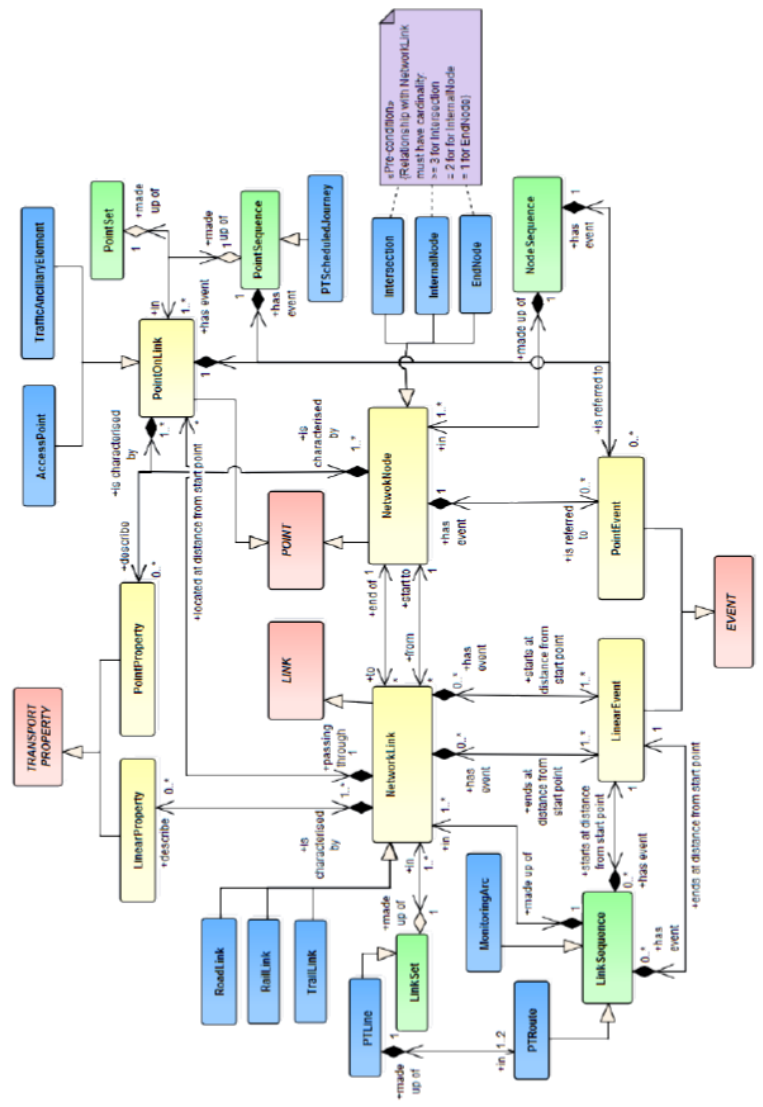

Figure 2. The conceptual data model

Between elements defined, a LinkSequence and a PointSequence represent an ordered list of objects (links or points) and it is used to define specific classes as: MonitoringArc (a group of links mainly used for traffic monitoring purposes), PTRoute (the linear path covered by a public transport vehicle in a specific direction of travel), PTScheduledJourney (ordered stop lists of a vehicle in a route, characterised by passing time). LinkSet and PointSet represent groups of elements without ordering rules. PointSet represent objects as group of access to the same public transport stop or point of interest, group of stops related to different services (bus, metro and train stops), group of traffic detectors managing a complex intersection. LinkSet are specialised in PTLine, which groups two PTRoute objects (composite relationship) in the opposite direction, known to the public by a common name or number.

The TRANSPORT PROPERTY abstract class is specialised in LinearProperty and PointProperty child classes. Those two classes are related with a composition association to their relevant spatial classes.

Finally, the EVENT abstract class is specialised in LinearEvent and PointEvent classes and related to their pertinent spatial object with a composition association, which can be single objects as NetworkNode, PointOnLink and NetworkLink, or grouping objects as LinkSequence and PointSequence. In addition, the LinearMeasure relationship with spatial element uses linear referencing method, which allows specifying a valid measure also along a partial stretch of the linear element. 


\subsection{The logical model}

The logical data model deepens the details of the conceptual data model in an application independent way, defining attributes and specifying relationships. Attention has been given to the definition of enumeration and code lists in order to build a robust semantic.

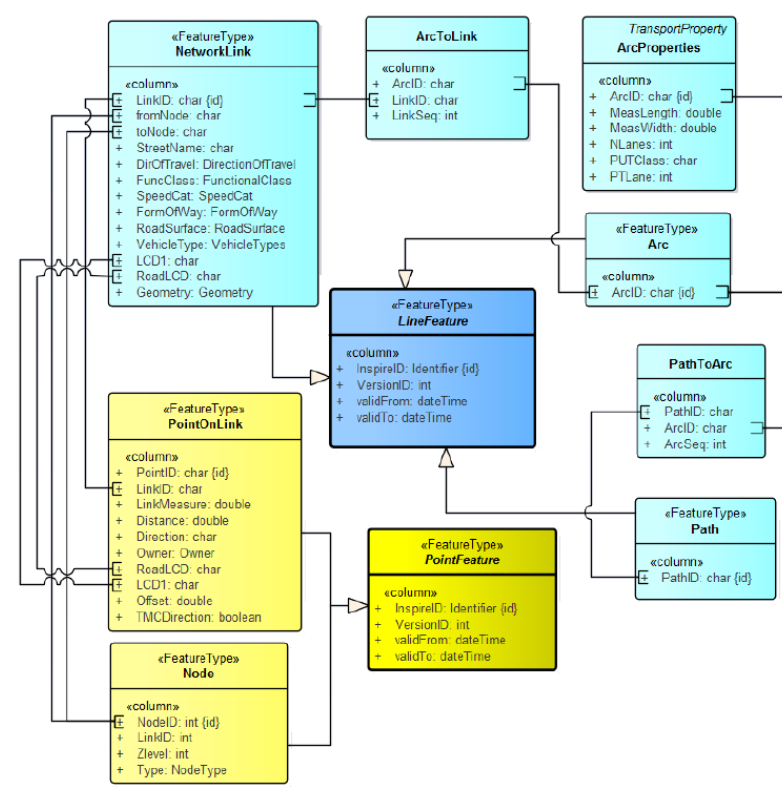

Figure 3. Diagram of road network elements.

As a first step, a common set of attributes that characterises all elements of the model have been defined. In particular, the field "InspireID" is global unique identifier, useful both for external publication purposes, and for version management; fields "validFrom", "validTo", and "VersionID" complete the set of fields to be used for version management. Even if most of classes have been defined as "FeatureType", a geometry field is explicitly added only for the class that represent linear road network, as all other elements can be referenced to it, and may optional have a spatial representation.

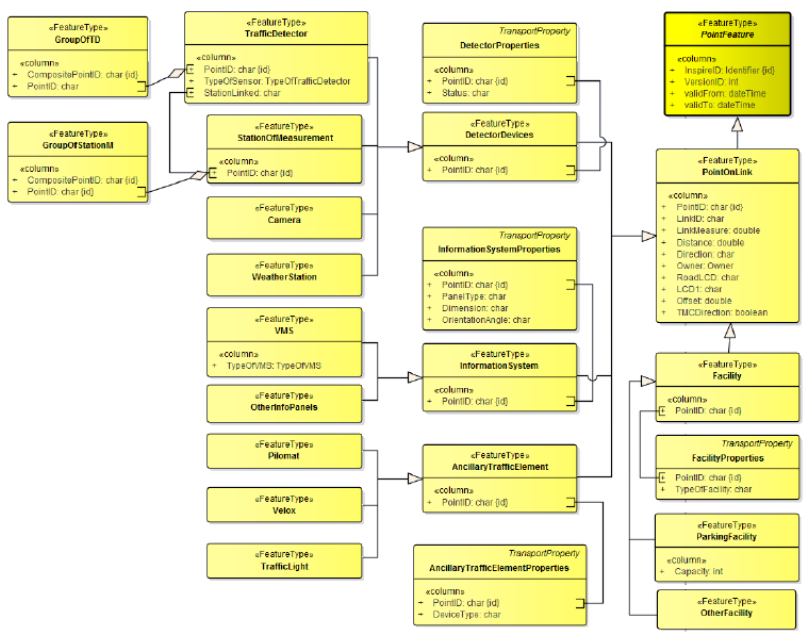

Figure 4: Diagram of ancillary traffic elements.

A diagram of classes involved in road segmentation model is shown in Figure 3. The NetworkLink class represents the linear road network and contains a set of attributes with associated enumeration values, which are mainly derived from INSPIRE Transport Network standard. Arc and Path also are a child of LineFeature class, representing an example of the LinkSequence/MonitoredArc class defined in the conceptual model. Arc is characterised by a set of properties defined by the external table ArcProperties (related through the field "ArcID"). The feature type Path groups together a series of Arc objects and is used mainly for traffic measures representation. In order to concretize the relationship between NetworkLink objects and LinkSequence, relationship tables have been defined: the ArcToLink table allows relating NetworkLink objects with Arc ones through the reference to identifiers, and, in the same way, the PathToArc table explicates the relationship between Arc and Path objects.

Looking at PointFeature child classes, the Node class represents elements used for line network definition. In particular, the field "Zlevel" describes the relative vertical position of the Node respect to the ground level, allowing to identify tunnels and bridges, and the field "Type" is used discriminate between intersections, internal and end nodes. The Node class is related to the NetworkLink class using the fields "fromNode" and "toNode" as foreign keys; additionally, Node class contains also the foreign key "LinkID", which identifies the reference link. This is enough to assure connectivity and define the direction of travel of the link.

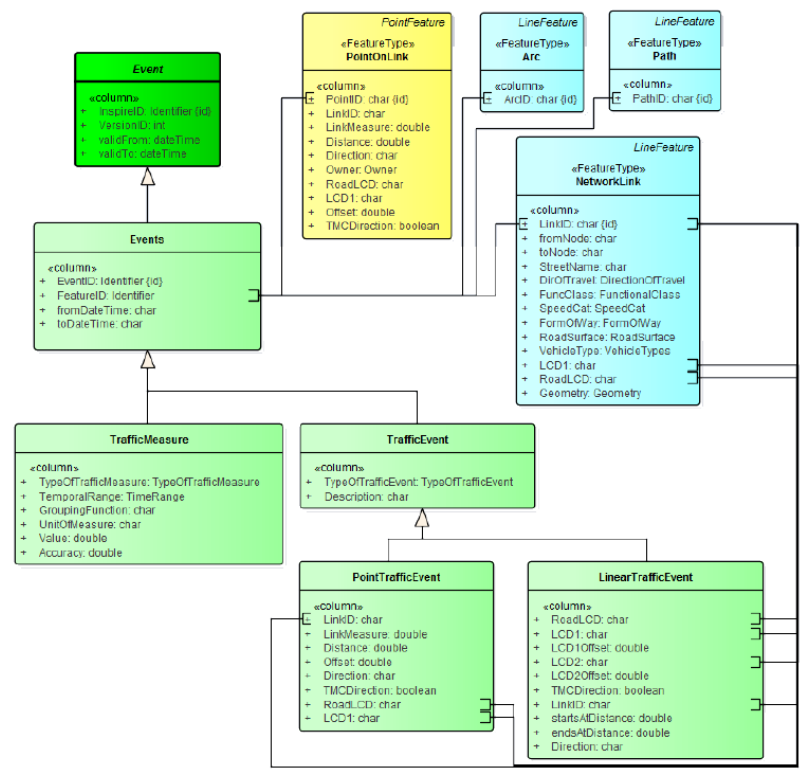

Figure 5: Diagram of events and relationships with network elements.

PointOnLink is the other child class of PointFeature and represents an object located along the linear network. Two linear location systems have been implemented: through the reference to the foreign key "LinkID" and the use of "LinkMeasure" and "Distance" fields is possible to locate the point along the link (as instance using ArcGIS linear referencing Toolbox). In addition, fields representing the TMC linear location systems can be used, implementing a custom linear referencing script. Both implementations have been considered as the TMC referencing is the most used for traffic events information spreading (e.g. through DATEX protocol), whereas other types of referencing allow to locate objects and events also on the lower hierarchy road network.

The PointOnLink class is further specialised in several classes, which represents the set of ancillary traffic elements that must be considered for traffic management (Figure 4). 
Finally, a set of elements derived from the Event abstract class is used for specifying traffic measures and events. A diagram showing classes and their relationships is shown in Figure 5.

The general Events class contains attributes useful to link data to spatial element ("FeatureID") and to define its temporal extent. In contrast with the previous classes, Events is always a table and needs to be linked to a spatial element or located through linear referencing in order to be visualised.

The child class TrafficMeasure has a set of attributes that allow to describe and characterise the type of measure. The other child class TrafficEvent is further specialised in order to define a point or linear event. In this case, in addition to the "FeatureID" attribute, also fields useful for TMC referencing method are defined. Indeed, a TrafficMeasure can be represented both as line or point, but it is usually referred to a defined PointOnLink, NetworkLink or groups of them. This is not true for traffic events, which can involves stretch of roads or located along a link (or link sequences): the TMC referencing enables this kind of visualisation. The associated enumeration TypeOfTrafficEvent allows distinguishing between several events and is based on the DATEX dictionary used in 5T. The "Description" field can be used to further describe the traffic event.

\section{RESULTS}

\subsection{Database implementation}

The physical implementation of the model is tailored on 5T exigences and set up with ArcGIS Geodatabase technology (Figure 6).

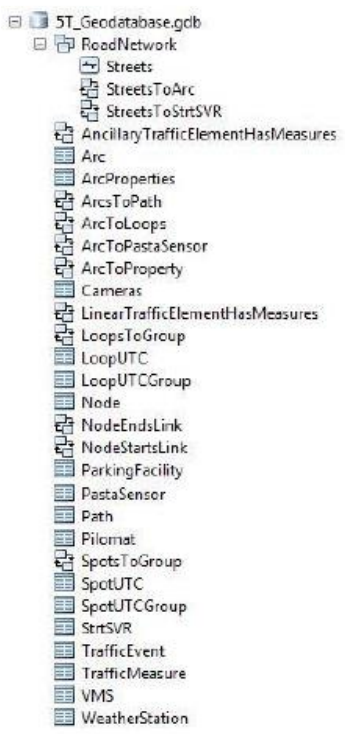

Figure 6: A view from ArcCatalog of the geodatabase

Compared to logical data model, enumerations have been converted in ArcGIS domains and associated to relevant fields. A series of subtypes manages the specialisation of ancillary traffic elements, and relationship classes manages aggregation relationship and connectivity rules. The geodatabase is composed by one feature dataset called RoadNetwork, which contains the polyline feature class Street, a customisation of the NAVSTREETS Streets Data, and the relationships for which it has a source role; all other elements are tables and so, located outside of the feature dataset.

In order to correctly load data into the data model, a broad activity of data transformation has been applied. Firstly, data relative to road network have been modified in order to find the matching between the different sources available in 5T (conflation activity between commercial datasets as NAVSTREETS Streets Data of 2014 and 2016 and 5T defined arcs and paths). Secondly, attributes have been mapped into new fields of the data model. Finally, a linear referencing activity has been performed in order to reduce the processing load when the geodatabase is used. Only after these steps, data have been loaded into the new geodatabase.

Last activity performed was the creation of custom scripts to extract and group measures and traffic events. The scripts for measures extraction perform basic queries (essentially a GROUP BY), giving as input the measure type, the start and end date time of interest, and optionally a predefined temporal range over which aggregate data, with a statistical function associated. This approach has been considered more efficient than storing predefined set of data that may overload the geodatabase. In this way, the computing power of the server is used for group by operation and fields transformation, whereas the machine has only to create the customised object class and the relationship class and load data into. Several scripts have been created in order to fulfil a set of possibility.

\subsection{Spatial and temporal applications}

As a first result, the geodatabase enables 5T opeartors to visualise the set of sensors and other traffic management element with the possibility to query and display only the needed subset with a customised symbology (Figure 7).

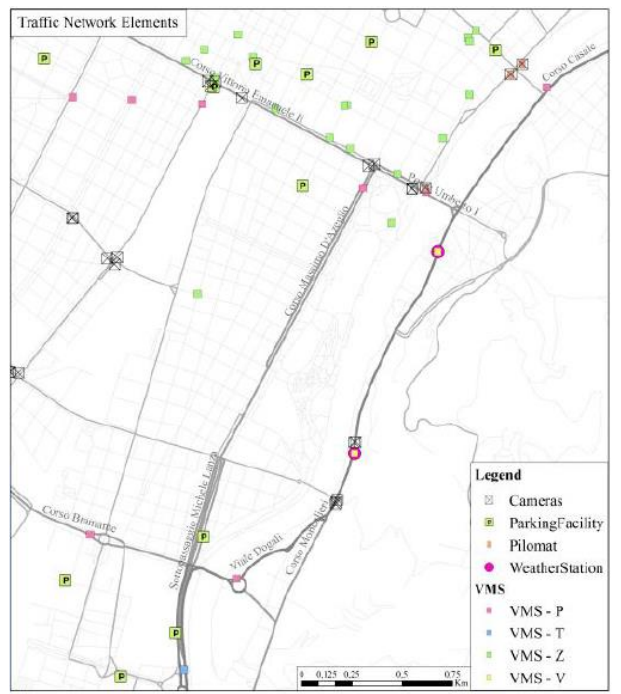

Figure 7. Position of other ancillary traffic elements.

This was previously quite complex in the past as the knowledge of data source location (separated in several servers) and the skills to extract data were not so accessible. In order to visualise point element position from the available tables, data need to be loaded in ArcMap and then using the "Display Route Events" interface data are visualised on the fly. Data can be exported as feature class or used as it with a customised symbolisation. Visualising Arcs and Path instead needs a further step: starting from the Street feature class, the "Join" interface must be used, selecting the option "Join data based on a pre-defined relationship class". In this way, fields of the related table are reported in the Street feature class and can be used for thematic visualisation.

The geodatabase schema defined allows the extraction of single measure on demand. As for the Arc and Path representation, 
once the feature table is load in ArcMap and data are visualised as Route Event, a join with the option "Join data based on a pre-defined relationship class" must be applied, linking data with measures. In Figure 8, a comparison of a set of measures detected by the UTC loops is given: first row represents the situation at a peak hour (h. 8:30), the second row represents the situation at a non-peak hour (h. 5:35). In this case, the measure is given as it is from sensors: no aggregation has been applied in data extraction procedure.

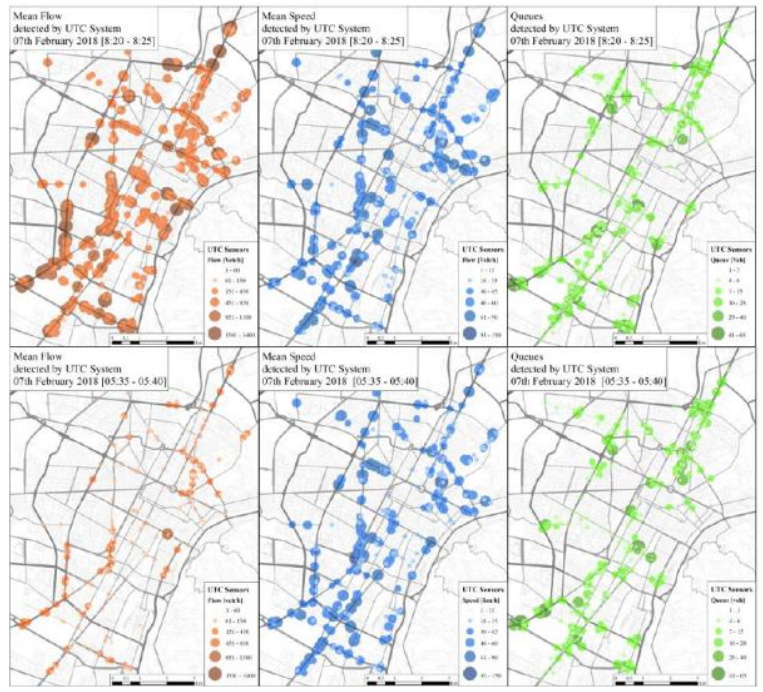

Figure 8: Traffic measures detected by UTC loops (flow, speed, queues), comparison of a single bin detection.

In a similar way, also measures relatives to Arc and Path can be visualised: in Figure 9, is shown the mean travel time measure (normalised on the free flow travel time measure), estimated by the SVM, and reported over Path features. As Arc (and consequently Path) can be referred to the same bidirectional link, the output visualisation shows Path elements overlay, with different measures referred to it. More appropriate rules of representation can be applied in order to not allow the overlay between measures, but in this case is quite effective to show the differences in traffic flow in opposite flow direction.

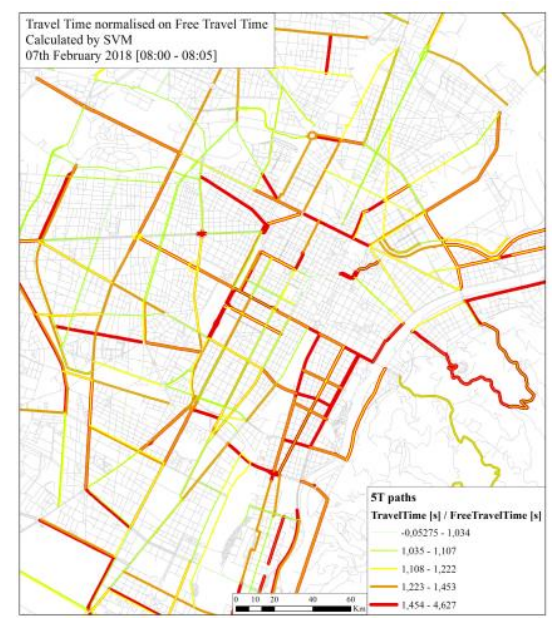

Figure 9. Travel time, normalised on free flow travel time, estimated by Metropolitan Supervisor (SVM).

Also, short time series can be represented though graphs. An example is given in Figure 10. In this case, in order to obtain the series, multiple extraction of the same measure has been done in sequential time ranges. Tables created are then joined together in order to obtain a single table to link with the relative spatial object. Then an appropriate symbolisation has been set. Finally, measures can also be integrated in a Network Dataset and used as real impedances. In Figure 11, the network dataset has been built on an export of Arc features, over which a table of measures has been previously joined. The figure shows the differences in routing choices made by the ArcGIS routing engine, setting different arc measures as impedance.

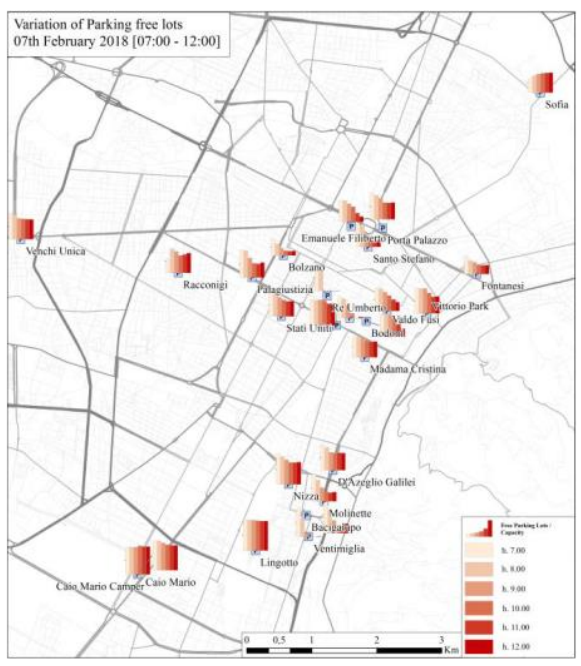

Figure 10. Variation of the availability of free lots in parking area, normalised on the capacity, in a time window (h. 7-12)

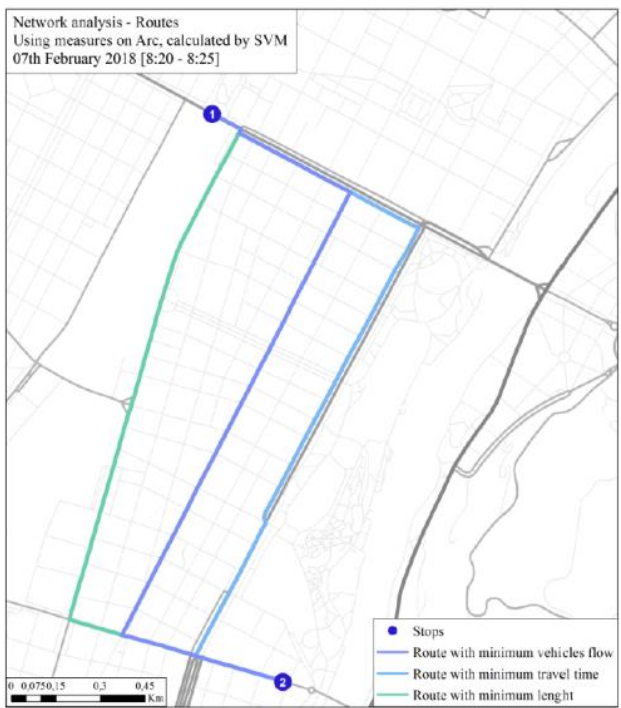

Figure 11. Differences in Route Analysis outputs using different measures of Arc as impedances.

\section{CONCLUSION}

The geodatabase proposed allows to easy extract, analyse and visualise the main physical infrastructure and their related objects, measures and properties, simplifying the production of reports on historical data as the finding of spatial and temporal correlation between traffic events. For the agency point of view, a strength of this result is the possibility to reuse data and measure for customized tasks, without the need to deeply know the entire ITS environment system which prop up the measures acquisition. 
This research has made evident some considerations: first of all, the difficult of use ITS data in GIS desktop solution in a straightforward way. The proposed solution tries to overcome the peculiarity of ITS platforms and concentrates on data available and mechanism to reuse ITS data in a GIS environment. From a spatial point of view, standard solution exists to manage data, but are sometimes too general, or related to a specific field of action. From the standard analysis has also emerged the difficult in creating a common dictionary of the terms involving the mobility: lot of terms are used for referring to the same concepts leading confusion when data integration is needed between TOCs. The analysed standards do not consider the representation of ancillary traffic elements as sensors, group of sensors, cameras, velox and traffic lights: the proposed data model indeed consider the representation of those elements and define their spatial relationships with the road network. In addition, it tries to develop general code lists (in the logical data model), implementing a first vocabulary activity which can be reused by other TOCs.

Looking at the logical data model proposed, as the solutions is not technology dependent, it can be used also by other TOCs, implementing it as whole or only partially and using the DBMS technology preferred. The proposed model indeed can be used also in an open source environment as PostgreSQL/PostGIS. Further developments will concern the set-up of the model for the whole Italy, within the context of the Smart Road Initiative, starting from the five cities (Turin, Milan, Padua, Rome, Naples) involved in the PRIN 2015 project URBAN-GEO BIG DATA.

\section{ACKNOWLEDGEMENTS}

URBAN-GEO BIG DATA (URBAN GEOmatics for Bulk Information Generation, Data Assessment and Technology Awareness) is a Project of National Interest (PRIN) funded by the Italian Ministry of Education, University and Research (MIUR) - id. 20159CNLW8.

\section{REFERENCES}

Arco E., Ajmar A., Arneodo F., Boccardo P., 2017. An operational framework to integrate traffic message channel (TMC) in emergency mapping services (EMS). In: European Journal of Remote Sensing, vol. 50-1, pp. 478-495. ISSN 22797254. DOI: $10.1080 / 22797254.2017 .1361306$.

Arneodo, F., Foti, G., Cocozza, M., 2009. S.I.MO.NE. Innovative system for metropolitan area mobility management. In: Proceedings of the 16th ITS World Congress, 21-25 September 2009, Stockholm, Sweden.

Butler, J.A., 2008. Designing geodatabase for transportation. Environmental Systems Research Institute, Inc., Redlands, CA, USA.

Cadkin, J., 2002 a. Dynamic Segmentation in ArcGIS. ArcUser, v.7, pp. 52 - 54. Available from: http://www.esri.com/news/arcuser/0702/files/dynseg.pdf September 2018).

Cadkin, J., 2002 b. Understanding Dynamic Segmentation Working With Events in ArcGIS 8.2. ArcUser, v.10, pp. $40-$ 43.

Available
http://www.esri.com/news/ArcUser/1002/files/dynseg_2.pdf (20 September 2018).

European Commission, 2011. WHITE PAPER Roadmap to a Single European Transport Area - Towards a competitive and resource efficient transport system. Available from: http://eurlex.europa.eu/legalcontent/EN/ALL/?uri=CELEX:52011DC014 4 (20 September 2018).

Federal Geographic Data Committee, 2008. Geographic Information Framework Data Content Standard. Federal Geographic Data Committee Secretariat, U.S. Geological Survey, Virginia, USA.

Goodwin, C., Latham, F. E., Siegel, D., Gordon, S., 1998. Intelligent Transportation Systems Datum Public Sector Requirements. Workshop Report in Spatial Data Interoperability Protocol for ITS Project, Oak Ridge National Laboratory, Oak Ridge, Tennessee.

INSPIRE, 2013. D2.10.1 INSPIRE Data Specifications - Base Models - Generic Network Model. Drafting Team "Data Specifications".

ISO, 2017. ISO in brief. Great Things Happen when the world agrees. Available from: https://www.iso.org/files/live/sites/isoorg/files/archive/pdf/en/is oinbrief_2015.pdf (20 September 2018)

Knowles, N., Miller, P., 2008. A Transmodel based XML schema for the Google Transit Feed Specification - With a GTFS / Transmodel comparison. Kizoom Ltd 109-123 Clifton Street, London. [online] Available from: http://naptan.dft.gov.uk/transmodel/schema/doc/GoogleTransit/ TransmodelForGoogle-09.pdf (20 September 2018)

Ministero delle Infrastrutture e dei Trasporti, 2018. Modalità attuative e strumenti operativi della sperimentazione su strada delle soluzioni di Smart Road e di guida connessa e automatica. Gazzetta Ufficiale della Repubblica Italiana, Serie generale, n.90. Available from: https://www.gazzettaufficiale.it/eli/id/2018/04/18/18A02619/sg (30 March 2019).

Rodrigue, J.P., 2017. The Geography of Transport Systems. Hofstra University, Dept. of Global Studies \& Geography, New York, USA. Available from: http://people.hofstra.edu/geotrans. (20 September 2018).

Thill, J.C., 2000. Geographic information systems for transportation in perspective. Transportation Research Part $C$ Emerging Technologies, v.8, pp. 3 - 12 .

Thompson, R., Wang, Y., Bishop, I., 2019. Integrating GIS with intelligent transport system and stochastic programming for improved vehicle scheduling. Available from: https://www.researchgate.net/publication/265410821 (30 March 2019).

TRANSMODEL, 2015. Data model in Enterprise Architect format. Available from: http://www.transmodelcen.eu/wpcontent/uploads/sites/2/Trm6-Part-1-2-3-

Sept2015.eap_.zip (20 September 2018) 